Siberian Mathematical Journal, Vol. 57, No. 1, pp. 74-85, 2016

Original Russian Text Copyright (c) 2016 Leı̆nartas E.K. and Nekrasova T.I.

\title{
CONSTANT COEFFICIENT LINEAR DIFFERENCE EQUATIONS ON THE RATIONAL CONES OF THE INTEGER LATTICE
}

\author{
E. K. Leĭnartas and T. I. Nekrasova
}

UDC $517.53 / 55$

\begin{abstract}
We obtain a sufficient solvability condition for Cauchy problems for a polynomial difference operator with constant coefficients. We prove that if the generating function of the Cauchy data of a homogeneous Cauchy problem lies in one of the classes of Stanley's hierarchy then the generating function of the solution belongs to the same class.
\end{abstract}

DOI: $10.1134 / \mathrm{S} 0037446616010080$

Keywords: higher-dimensional difference equations, Cauchy problem, generating function, $D$-finite Laurent series

\section{Introduction}

Define on the complex-valued functions $f(x)=f\left(x_{1}, \ldots, x_{n}\right)$ of integer variables $x_{1}, \ldots, x_{n}$ the shift operators $\delta_{j}$ with respect to $x_{j}$ as $\delta_{j} f(x)=f\left(x_{1}, \ldots, x_{j-1}, x_{j}+1, x_{j+1}, \ldots, x_{n}\right)$ and the polynomial difference operators

$$
P(\delta)=\sum_{\omega \in \Omega} c_{\omega} \delta^{\omega}
$$

where $\Omega \subset \mathbb{Z}^{n}$ is a finite set of points in the $n$-dimensional integer lattice $\mathbb{Z}^{n}$ and $\delta^{\omega}=\delta_{1}^{\omega_{1}} \cdot \ldots \cdot \delta_{\omega}^{\omega_{n}}$, while $c_{\omega}$ are constant coefficients of the difference operator.

Consider the difference equations

$$
P(\delta) f(x)=g(x), \quad x \in X,
$$

where $f(x)$ is an unknown function, while $g(x)$ is a prescribed function on some fixed set $X \subset \mathbb{Z}^{n}$. Select in $X$ a subset $X_{0} \subset X$ of points called initial (boundary) points and state the following problem.

Find a function $f(x)$ satisfying (1) and coinciding on $X_{0}$ with some specified function $\varphi(x)$ :

$$
f(x)=\varphi(x), \quad x \in X_{0} .
$$

It is natural to refer to this problem as a Cauchy problem for (1), while to the function $\varphi(x)$ in $(2)$ as the Cauchy data of (1). The existence and uniqueness of a solution to a Cauchy problem depend on all objects involved in its statement: the difference operator $P(\delta)$, the set $X$ on which the right-hand side $g(x)$ of (1) is defined, and $X_{0}$ on which the Cauchy data $\varphi(x)$ are given. There are no general results concerning the relations between these objects to ensure the existence and uniqueness of solutions to Cauchy problems, and they seem hard to describe. By way of example, the discretization of the equations of mathematical physics using the methods of scheme theory leads to diverse problems of the form (1)-(2) in which the choice of $X$ and $X_{0}$ depends on the differential problem (see [1] for instance). The discretization of Cauchy-Riemann equations spawned the theory of discrete analytic and harmonic functions [2]. Some fruitful combinatorial ideas were contributed to it in $[3,4]$.

This research was done at Siberian Federal University and supported by the Government of the Russian Federation (Grant 14.Y26.31.0006). The first author was also supported by the Russian Foundation for Basic Research (Grant 14-01-00-544).

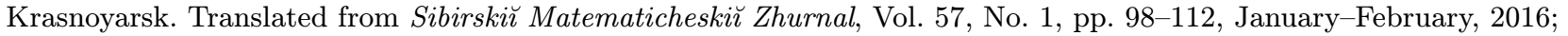
DOI: 10.17377/smzh.2016.57.108. Original article submitted November 10, 2014. 
We are interested in certain problems of the form (1)-(2) which arise in combinatorial analysis. In the one-dimensional case the difference operator is of the form $P(\delta)=\sum_{\omega=0}^{m} c_{\omega} \delta_{\omega}$ with $c_{m} \neq 0$. As the set $X$ on which the right-hand side is defined and a solution $f(x)$ to (1) is sought we take the set $\mathbb{Z}_{+}$of nonnegative integers, and as $X_{0}$ we take $X_{0}=\{0,1, \ldots, m-1\}$. Under these assumptions problem (1)-(2) obviously has the unique solution. In many cases the standard situation is of $X=\mathbb{Z}_{+}^{n}$, while the choice of $X_{0}$ depends on the properties of the set $\Omega$ defining $P$. For the difference equation (1) the question of "correct" statement (i.e., ensuring the existence and uniqueness of a solution) of the Cauchy problem in the positive octant $\mathbb{Z}_{+}^{n}$ of the integer lattice was studied in [5] together with the algebraic nature of the generating function for the solution to the difference equation. In this article we address these questions in a more general situation; namely, instead of the positive octant $\mathbb{Z}_{+}^{n}$ we take the intersection of a rational cone $K$ with $\mathbb{Z}^{n}$, which we call a rational cone of the integer lattice. Let us give necessary notations and definitions.

Given linearly independent vectors $a^{1}, \ldots, a^{n}$, where $a^{j}=\left(a_{1}^{j}, \ldots, a_{n}^{j}\right)$, with integer coordinates $a_{i}^{j} \in \mathbb{Z}$, refer as the rational cone generated by $a^{1}, \ldots, a^{n}$ to the set

$$
K=\left\{x \in \mathbb{R}^{n}: x=\lambda_{1} a^{1}+\cdots+\lambda_{n} a^{n}, \lambda_{j} \in \mathbb{R}_{+}, j=1, \ldots, n\right\} .
$$

Note that $K$ is a simplicial cone; i.e., each element of $K$ has the unique representation via generators. Also, $K$ is a salient cone, i.e., $K$ contains no lines.

Define the partial order $\underset{K}{\geq}$ on the points $u, v \in \mathbb{R}^{n}$ as follows:

$$
u \underset{K}{\geq} v \Leftrightarrow u \in v+K
$$

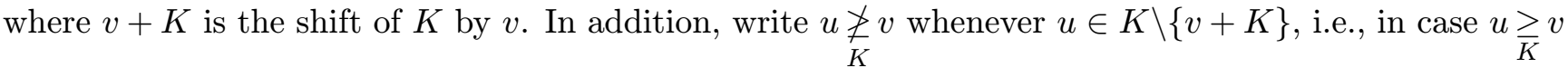
is false.

Fix $m \in \Omega$ and make problem (1)-(2) more concrete: Take as $X$ the rational cone $K \cap \mathbb{Z}^{n}$ of the

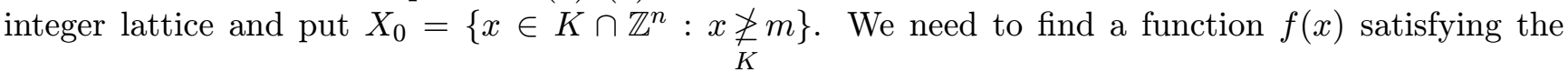
equation

$$
P(\delta) f(x)=g(x), \quad x \in K \cap \mathbb{Z}^{n},
$$

and coinciding with a specified function $\varphi(x)$ on $X_{0}$ :

$$
f(x)=\varphi(x), \quad x \in X_{0} .
$$

Let us state a condition on the difference operator $P(\delta)$ ensuring the existence and uniqueness of a solution to (3)-(4).

The dual cone to $K$ is

$$
K^{*}=\left\{k \in \mathbb{R}^{n}:\langle k, x\rangle \geq 0, x \in K\right\},
$$

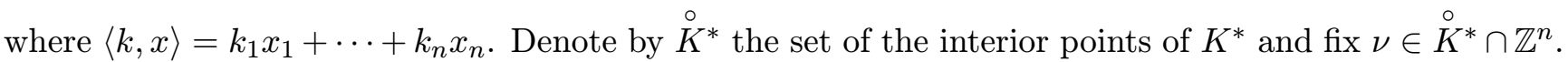
For all $x \in K \cap \mathbb{Z}^{n}$ refer as the weighted homogeneous power of a monomial $z^{x}$ to the nonnegative number $|x|_{\nu}=\langle\nu, x\rangle$ and define the (weighted homogeneous) degree of the Laurent polynomial $Q(z)=\sum_{x} q_{x} z^{x}$ as $\operatorname{deg}_{\nu} Q(z)=\max _{x}|x|_{\nu}$.

Denote by $\mathbb{C}_{K}[z]$ the ring of Laurent polynomials $Q(z)=\sum_{x} q_{x} z^{x}$ with the exponents $x$ of the monomials $z^{x}$ lying in $K \cap \mathbb{Z}^{n}$. Addition and multiplication are defined naturally.

Refer to the Laurent polynomial $P(z)=\sum_{\omega \in \Omega} c_{\omega} z^{\omega}$ as the characteristic polynomial of the difference equation (3). Refer as the order of $P(\delta)$ to the degree $\operatorname{deg} P(z)$ of the characteristic polynomial. Denoting this order by $d$, we can express $P(\delta)$ as

$$
P(\delta)=\sum_{|\omega|_{\nu} \leq d} c_{\omega} \delta^{\omega} .
$$


Theorem 1. If for the point $m$ defining $X_{0}$ of Cauchy data there is $\nu \in \stackrel{\circ}{K^{*} \cap} \mathbb{Z}^{n}$ such that $|m|_{\nu}=d$ and $m$ is the unique point in $\Omega$ with these properties then (3)-(4) has the unique solution.

For $n=1$ it is known that the generating function of the solution to the difference equation with arbitrary Cauchy data is rational, whereas for $n>1$ that is false. For difference equations in the positive octant $\mathbb{Z}_{+}^{n}$ of the integer lattice, an example in [5] shows that the rationality of the generating function of Cauchy data fails in general to imply the rationality of the generating function of the solution, which need not even be $D$-finite. Some conditions on the equation are also given which ensure the rationality (algebraicity) of the generating functions of solutions in the case of rational (algebraic) generating functions of Cauchy data.

Refer as the generating function (series) of a function $f: K \cap \mathbb{Z}^{n} \rightarrow \mathbb{C}$ of integer arguments $x=$ $\left(x_{1}, \ldots, x_{n}\right)$ to the Laurent series

$$
F(z)=\sum_{x \in K \cap \mathbb{Z}^{n}} f(x) z^{-x}
$$

The series of this form constitute the ring $\mathbb{C}_{K}[[z]]$ under addition and multiplication. The multiplication of two series is defined in the usual way and is well-defined because $K$ is a salient cone. In particular, this means that the number of expressions of each vector $\varrho \in K \cap \mathbb{Z}^{n}$ as $\varrho=x+y$ with $x, y \in K \cap \mathbb{Z}^{n}$ is finite.

The most useful classes of generating functions (power series) in enumerative combinatorial analysis form the hierarchy proposed by Stanley (see [6]):

$$
\{\text { rational }\} \subset\{\text { algebraic }\} \subset\{D \text {-finite }\} .
$$

There arises the question whether the generating functions (series) for the solution to a difference equation remain in the same class of this hierarchy as the generating functions of Cauchy data. The solvability conditions for problem (3)-(4) in Theorem 1 are insufficient to give the positive answer to this question (see $[5])$.

Impose the stronger condition: The point $m$ specifying the set $X_{0}$ on which the Cauchy data of problem (3)-(4) are given must satisfy the relation

$$
m \underset{K}{\geq} \omega \text { for all } \omega \in \Omega \text {. }
$$

Under this condition, for the generating function

$$
F(z)=\sum_{x \in K \cap \mathbb{Z}^{n}} f(x) z^{-x}
$$

of the solution $f(x)$ to the homogeneous problem (3)-(4) we have the formula (see Theorem 3 of [2])

$$
F(z)=\sum_{\omega \in \Omega} c_{\omega} z^{\omega} \frac{1}{P(z)} \Phi_{\omega}(z),
$$

where

$$
\Phi_{\omega}(z)=\sum_{x \in K \cap \mathbb{Z}^{n}, x \underset{K}{\ngtr \omega}} \varphi(x) z^{-x} .
$$

Condition (6) implies that the generating function $\Phi(z)$ of the Cauchy data equals $\sum_{x \in X_{0}} \varphi(x) z^{-x}=$ $\Phi_{m}(z)$. By (7), for the rationality or algebraicity of the generating function $F(z)$ the rationality or algebraicity of $\Phi_{\omega}(z)$ for all $\omega \in \Omega$ is sufficient. As for $D$-finiteness, we need firstly to define this concept for Laurent series supported on a rational cone of the integer lattice. To this end, define the operators that, in contrast to the partial derivatives, are derivations of $\mathbb{C}_{K}[[z]]$. 
We can express each element $x \in K \cap \mathbb{Z}^{n}$ as a linear combination $x=\lambda_{1} a^{1}+\cdots+\lambda_{n} a^{n}$ of basis vectors. In matrix form this expression becomes $x=A \lambda$, where $\lambda$ is a column vector, and $A$ is the matrix with the coordinates of the vectors $a^{j}$ in columns,

$$
A=\left(\begin{array}{ccc}
a_{1}^{1} & \ldots & a_{1}^{n} \\
& \ldots & \\
a_{n}^{1} & \ldots & a_{n}^{n}
\end{array}\right) .
$$

The determinant $\Delta$ of $A$ is nonzero.

Define the operator $D_{i}$ on the monomials $f(x) z^{-x}$ as follows:

$$
D_{i} f(x) z^{-x}=-\lambda_{i} f(x) z^{-x-a^{i}},
$$

where $\lambda_{i}$ is the $i$ th coordinate of the point $\lambda=A^{-1} x$. Observe that for $\Delta \neq 1$ and $x \in K \cap \mathbb{Z}^{n}$ the number $\lambda_{i}$ is rational in general.

The action of $D_{i}$ on a series $F$ in $\mathbb{C}_{K}[[z]]$ is defined by linearity. It is not difficult to verify that $D_{i}$ for $i=1, \ldots, n$ are derivations (i.e., they map $\mathbb{C}_{K}[[z]]$ into itself, are linear, and satisfy the usual Leibniz product rule for the derivative). Put $D_{i}^{k}=\underbrace{D_{i} \circ \cdots \circ D_{i}}_{k \text { times }}$.

Definition 1. A formal series

$$
F(z)=\sum_{x \in K \cap \mathbb{Z}^{n}} f(x) z^{-x}
$$

in the ring $\mathbb{C}_{K}[[z]]$ is called $D$-finite whenever it satisfies a system of equations

$$
Q_{k}^{i}(z) D_{i}^{k} F(z)+\cdots+Q_{1}^{i}(z) D_{i} F(z)+Q_{0}^{i}(z) F(z)=0, \quad i=1, \ldots, n,
$$

with $Q_{j}^{i}(z) \in \mathbb{C}_{K}[z]$.

If $K=\mathbb{R}_{+}^{n}$ then $D_{i}=\frac{\partial}{\partial z_{i}}$ and we recover Lipshitz's definition of $D$-finiteness of power series (see [7]).

Theorem 2. If the point $m$ defining the set $X_{0}$ on which the Cauchy data of the homogeneous problem (3)-(4) are given satisfies (6) then the $D$-finiteness (rationality or algebraicity) of the generating function of the Cauchy data implies the $D$-finiteness (rationality or algebraicity) of the generating function of the solution.

It is clear from (7) that in order to establish the $D$-finiteness (rationality or algebraicity) of the generating function $F(z)$ of the solution to the homogeneous problem (3)-(4) it suffices to show that the product of $D$-finite series is $D$-finite and that the $D$-finiteness (rationality or algebraicity) of the generating function of the Cauchy data $\Phi \in \mathbb{C}[[z]]$ implies that the series $\Phi_{\omega}$ is $D$-finite (rational or algebraic) for all $\omega \in K \cap \mathbb{Z}^{n}$. We do this in Propositions 4 and 5 of Section 5 . Their proofs rely respectively on two available properties of $D$-finite power series:

The $D$-finite series constitute a subalgebra in the algebra of power series;

The sections of $D$-finite (rational or algebraic) power series are $D$-finite (rational or algebraic).

\section{Solvability of the Cauchy Problem}

In this section we prove Theorem 1 on the solvability of (3)-(4). The problem amounts to an infinite system of equations in infinitely many variables $f(x)$ with $x \in K \cap \mathbb{Z}^{n}$ of a specific form, as only finitely many unknowns appear in each equation. This system is consistent provided that so is every subsystem of finitely many equations (see [8, Chapter 6, Lemma 6.3.7] for instance). Below we construct a sequence of subsystems of (3)-(4) with finitely many equations. Each of these subsystems includes all equations of the previous one. By the lemma mentioned above, the consistency of each of them implies the consistency of (3)-(4). 
Introduce the relation $\underset{K}{\prec}$ on the integer points of the rational cone $K$. Denote by $\prec$ the lexicographic order on $\mathbb{Z}_{+}^{n}$. Then define the relation $\underset{K}{\prec}$ for $x, y \in K \cap \mathbb{Z}^{n}$ as follows:

$$
x \underset{K}{\prec} y \Leftrightarrow \Delta A^{-1} x \prec \Delta A^{-1} y,
$$

where $A^{-1}$ is the inverse matrix to $A$, and $\Delta=\operatorname{det} A$.

For a vector $\nu$ chosen in the conditions of Theorem 1 consider the linear function $\langle\nu, x\rangle$ of $x \in K$. Order the set of its values at the points of $K \cap \mathbb{Z}^{n}$ and denote it by $S$. Observe that $S \subset \mathbb{Z}_{+}$since $\nu \in \stackrel{\circ}{K^{*}}$. Define the weighted lexicographic order $\triangleleft$ on the set of integer points of the cone $K$ as follows: for $x, y \in K \cap \mathbb{Z}^{n}$ the relation $x \triangleleft y$ means that $\langle\nu, x\rangle<\langle\nu, y\rangle$, while if $\langle\nu, x\rangle=\langle\nu, y\rangle$ then $x \prec y$.

Take some $s \in S$. Enumerate the unknowns by the elements of $J_{s}=\left\{y \in K \cap \mathbb{Z}^{n}:\langle\nu, y\rangle \leq s\right\}$, and the equations by the elements of the sets

$$
I_{s}=\left\{x \in K \cap \mathbb{Z}^{n}:\langle\nu, x\rangle \leq s-\langle\nu, m\rangle\right\}, \quad I_{m, s}=\left\{\mu \in X_{0}:\langle\nu, \mu\rangle \leq s\right\} .
$$

Assign to the points $x$ of $I_{s}$ the "indices" of the points $m+x \in J_{s}$. It is not difficult to see that $\# I_{s}+\# I_{m, s}=\# J_{s}$, where $\# M$ stands for the size of a finite set $M$. Thus, we obtain a system of linear equations in the unknowns $f(y)$ for $y \in J_{s}$ of the form

$$
\begin{gathered}
\sum_{\omega \in \Omega} c_{\omega} f(x+\omega)=g(x), \quad x \in I_{s}, \\
f(\mu)=\varphi(\mu), \quad \mu \in I_{m, s} .
\end{gathered}
$$

Denote by $\Delta_{m, s}$ the determinant of (10)-(11).

By way of example, look at (10)-(11) in a version of the problem of generalized Dyck paths. Take the cone generated by $(1,1)$ and $(1,-1)$ and consider the difference equation

$$
f\left(x_{1}+2, x_{2}\right)+c_{1} f\left(x_{1}, x_{2}\right)+c_{2} f\left(x_{1}+1, x_{2}-1\right)+c_{3} f\left(x_{1}+1, x_{2}\right)+c_{4} f\left(x_{1}+1, x_{2}+1\right)=0
$$

for $x \in K \cap \mathbb{Z}^{2}$. In this case $\Omega=\{(2,0),(0,0),(1,-1),(1,0),(1,1)\}$. Take $m=(2,0)$ and seek the solution to the equations which agrees with the Cauchy data

$$
f\left(x_{1}, x_{2}\right)=\varphi\left(x_{1}, x_{2}\right), \quad\left(x_{1}, x_{2}\right) \in X_{0},
$$

where $X_{0}=\left\{\left(\mu_{1}, \mu_{2}\right) \in K \cap \mathbb{Z}^{2}:\left(\mu_{1}, \mu_{2}\right) \underset{K}{\nsupseteq}(2,0)\right\}$.

For $s=3$ the system (10)-(11) becomes

$$
\begin{aligned}
f\left(x_{1}+2, x_{2}\right)+ & c_{1} f\left(x_{1}, x_{2}\right)+c_{2} f\left(x_{1}+1, x_{2}-1\right)+c_{3} f\left(x_{1}+1, x_{2}\right) \\
& +c_{4} f\left(x_{1}+1, x_{2}+1\right)=0, \quad x \in I_{3}, \\
& f\left(\mu_{1}, \mu_{2}\right)=\varphi\left(\mu_{1}, \mu_{2}\right), \quad \mu \in I_{(2,0), 3},
\end{aligned}
$$

where the set

$$
\begin{gathered}
J_{3}=\{(0,0),(1,-1),(1,0),(1,1),(2,-2),(2,-1),(2,0),(2,1),(2,2), \\
(3,-3),(3,-2),(3,-1),(3,0),(3,1),(3,2),(3,3)\}
\end{gathered}
$$

enumerates the unknowns $f\left(x_{1}, x_{2}\right)$, while we also have

$$
\begin{gathered}
I_{(2,0), 3}=\{(0,0),(1,-1),(1,0),(1,1),(2,-2),(2,-1), \\
(2,1),(2,2),(3,-3),(3,-2),(3,2),(3,3)\}
\end{gathered}
$$


and $I_{3}=\{(\overline{0}, \overline{0}),(\overline{1},-\overline{1}),(\overline{1}, \overline{0}),(\overline{1}, \overline{1})\}$. The bar over the coordinates of a point $\left(\bar{x}_{1}, \bar{x}_{2}\right)$ means that this point has the same index as $\left(2+x_{1}, x_{2}\right)$. Then the determinant of the matrix of the system (12)-(13) has the form

$$
\Delta_{(2,0), 3}=\left|\begin{array}{cccccccccccccccc}
1 & 0 & 0 & 0 & 0 & 0 & 0 & 0 & 0 & 0 & 0 & 0 & 0 & 0 & 0 & 0 \\
0 & 1 & 0 & 0 & 0 & 0 & 0 & 0 & 0 & 0 & 0 & 0 & 0 & 0 & 0 & 0 \\
0 & 0 & 1 & 0 & 0 & 0 & 0 & 0 & 0 & 0 & 0 & 0 & 0 & 0 & 0 & 0 \\
0 & 0 & 0 & 1 & 0 & 0 & 0 & 0 & 0 & 0 & 0 & 0 & 0 & 0 & 0 & 0 \\
0 & 0 & 0 & 0 & 1 & 0 & 0 & 0 & 0 & 0 & 0 & 0 & 0 & 0 & 0 & 0 \\
0 & 0 & 0 & 0 & 0 & 1 & 0 & 0 & 0 & 0 & 0 & 0 & 0 & 0 & 0 & 0 \\
c_{1} & c_{2} & c_{3} & c_{4} & 0 & 0 & 1 & 0 & 0 & 0 & 0 & 0 & 0 & 0 & 0 & 0 \\
0 & 0 & 0 & 0 & 0 & 0 & 0 & 1 & 0 & 0 & 0 & 0 & 0 & 0 & 0 & 0 \\
0 & 0 & 0 & 0 & 0 & 0 & 0 & 0 & 1 & 0 & 0 & 0 & 0 & 0 & 0 & 0 \\
0 & 0 & 0 & 0 & 0 & 0 & 0 & 0 & 0 & 1 & 0 & 0 & 0 & 0 & 0 & 0 \\
0 & 0 & 0 & 0 & 0 & 0 & 0 & 0 & 0 & 0 & 1 & 0 & 0 & 0 & 0 & 0 \\
0 & c_{1} & 0 & 0 & c_{2} & c_{3} & c_{4} & 0 & 0 & 0 & 0 & 1 & 0 & 0 & 0 & 0 \\
0 & 0 & c_{1} & 0 & 0 & c_{2} & c_{3} & c_{4} & 0 & 0 & 0 & 0 & 1 & 0 & 0 & 0 \\
0 & 0 & 0 & c_{1} & 0 & 0 & c_{2} & c_{3} & c_{4} & 0 & 0 & 0 & 0 & 1 & 0 & 0 \\
0 & 0 & 0 & 0 & 0 & 0 & 0 & 0 & 0 & 0 & 0 & 0 & 0 & 0 & 1 & 0 \\
0 & 0 & 0 & 0 & 0 & 0 & 0 & 0 & 0 & 0 & 0 & 0 & 0 & 0 & 0 & 1
\end{array}\right| .
$$

Proof of Theorem 1. The algorithm for ordering the unknowns and the equations of (10)-(11) implies that in the unit rows of the determinant $\Delta_{m, s}$ corresponding to the equations of (11), all elements vanish but one equal to 1 on the main diagonal. As for the rows of the determinant corresponding to the equations of (10), firstly, the only nonvanishing elements are $c_{\omega}$ and, secondly, the hypotheses of Theorem 1 yield $\omega \triangleleft m$ for $\omega \in \Omega$ with $\omega \neq m$; hence, $x+\omega \triangleleft x+m$. Therefore, in the row of the determinant corresponding to (10) the last nonvanishing element is $c_{m}$. Since the equation has the index $x+m$, which equals the index of the unknown $y$, it follows that $c_{m}$ appears on the main diagonal. Thus, $\Delta_{m, s}$ is the determinant of a lower-triangular matrix with nonzero elements $c_{m}$ on the main diagonal; i.e., $\Delta_{m, s} \neq 0$ for all $s \in S$.

\section{The Generating Function of the Solution to the Cauchy Problem}

In this section we find a formula expressing the generating function of the solution in terms of the generating function of the Cauchy data.

We need some facts of the theory of amoebas of algebraic hypersurfaces (see [9]). Denote by $V=$ $\left\{z \in \mathbb{C}^{n}: P(z)=0\right\}$ the hypersurface of zeros of the characteristic polynomial $P(z)$. The amoeba $\mathscr{A}_{V}$ of the characteristic hypersurface $V$ is the image of $V$ under the mapping

$$
\log : z=\left(z_{1}, \ldots, z_{n}\right) \rightarrow\left(\log \left|z_{1}\right|, \ldots, \log \left|z_{n}\right|\right) .
$$

The Newton polytope $N_{P}$ of $P(z)$ is the convex hull in $\mathbb{R}^{n}$ of the elements of $\Omega$.

The complement of the amoeba $\mathbb{R}^{n} \backslash \mathscr{A}_{V}$ consists of finitely many open connected components $\{E\}$. In addition, we have the injective mapping $\sigma:\{E\} \rightarrow N_{P} \cap \mathbb{Z}^{n}$ such that \# vert $N_{P} \leq \#\{E\} \leq \# N_{P} \cap \mathbb{Z}^{n}$. In particular, every vertex $m$ of the Newton polytope $N_{P}$ corresponds to the component $E_{m}$ of the complement of the amoeba, to which we can associate the expansion of the function $\frac{1}{P(z)}$ as the Laurent series

$$
\frac{1}{P(z)}=\sum_{x} \frac{\mathscr{P}_{m}(x)}{z^{x}},
$$

where

$$
\mathscr{P}_{m}(x)=\frac{1}{(2 \pi i)^{n}} \int_{\Gamma} \frac{\xi^{x}}{P(\xi)} \frac{d \xi}{\xi},
$$

while $\Gamma=\log ^{-1} u$ for $u \in E_{m}$, and the Laurent series (14) converges in $\log ^{-1} E_{m}$.

The support of (5) is the set $\operatorname{supp} F=\left\{x \in K \cap \mathbb{Z}^{n}: f(x) \neq 0\right\}$. 
Proposition 1. If the point $m$ defining the set $X_{0}$ on which the Cauchy data of problem (3)-(4) are given satisfies (6) then the support of the series (14) lies in the cone $K$; i.e., $\frac{1}{P(z)} \in \mathbb{C}_{K}[[z]]$.

Proof. Condition (6) implies that $m$ is a vertex of the polytope $N_{P}$; hence, the function $\frac{1}{P(z)}$ decomposes as a series of the form (14) converging on the nonempty set $\log ^{-1} E_{m}$, while we can obtain the coefficients $\mathscr{P}_{m}(x)$ of $(14)$ as follows: At the first step use the expansion of a geometric progression:

$$
\frac{1}{P(z)}=\frac{1}{c_{m} z^{m}+\sum_{\omega \neq m} c_{\omega} z^{\omega}}=\frac{1}{c_{m} z^{m}\left(1-\sum_{\omega \neq m} \tilde{c}_{\omega} z^{\omega-m}\right)}=\frac{1}{c_{m} z^{m}} \sum_{k=0}^{\infty}\left(\sum_{\omega \neq m} \tilde{c}_{\omega} z^{\omega-m}\right)^{k}
$$

and then, upon standard rearrangements, arrive at an expansion of the form

$$
\frac{1}{P(z)}=\sum_{x \in m+K_{1} \cap \mathbb{Z}^{n}} \frac{\mathscr{P}_{m}(x)}{z^{x}}
$$

where $K_{1}$ is generated by the vectors $\omega-m$ for $\omega \in \Omega$; thus, $K_{1} \subset K \cap \mathbb{Z}^{n}$.

Theorem 3. If a vertex $m$ of the Newton polytope $N_{P}$ satisfies (6) then

(1) for the generating function of the solution $f(x)$ to the homogeneous problem (3)-(4) we have

$$
F(z)=\sum_{\omega \in \Omega} c_{\omega} z^{\omega} \frac{1}{P(z)} \Phi_{\omega}(z)
$$

where by $\frac{1}{P(z)}$ we understand the series expansion of this function supported by the affine cone $m+K$ and converging on $\log ^{-1} E_{m}$;

(2) for the solution $f(x)$ to the homogeneous problem (3)-(4) we have

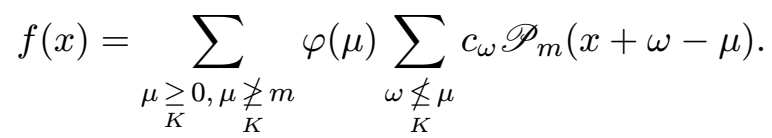

Proof. (1) Multiply the generating function

$$
F(z)=\sum_{x \in K \cap \mathbb{Z}^{n}} \frac{f(x)}{z^{x}}
$$

of the solution to (3)-(4) by the characteristic polynomial $P(z)$ and rearrange the product considering that $f(x)$ is a solution to (3), while $\varphi(x)$ is the Cauchy data of the Cauchy problem

$$
\begin{aligned}
& P(z) F(z)=\left(\sum_{\omega \in \Omega} c_{\omega} z^{\omega}\right)\left(\sum_{x \in K \cap \mathbb{Z}^{n}} \frac{f(x)}{z^{x}}\right)=\sum_{\omega \in \Omega} c_{\omega} z^{\omega}\left(\sum_{\substack{x \geq \omega \\
K}} \frac{f(x)}{z^{x}}+\sum_{x \geq \underset{K}{\omega}} \frac{f(x)}{z^{x}}\right)
\end{aligned}
$$

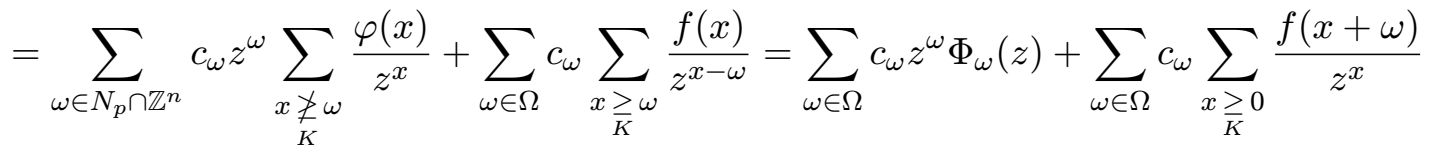

$$
\begin{aligned}
& =\sum_{\omega \in \Omega} c_{\omega} z^{\omega} \Phi_{\omega}(z)+\sum_{x \geq 0} \sum_{\bar{K}} \frac{c_{\omega} f(x+\omega)}{z^{x}}=\sum_{\omega \in \Omega} c_{\omega} z^{\omega} \Phi_{\omega}(z) .
\end{aligned}
$$

Thus, $P(z) F(z)=\sum_{\omega \in \Omega} c_{\omega} z^{\omega} \Phi_{\omega}(z)$, and upon multiplying by (14) we justify claim (1) of Theorem 3 . 
(2) The supports of the series $\frac{1}{P(z)}$ and $\Phi_{\omega}(z)$ lie in $K \cap \mathbb{Z}^{n}$. Multiply these series and collect similar terms on the right-hand side of (15). Equating the coefficients of the resulting series and the coefficients $f(x)$ of the series expansion of $F(z)$, we obtain (16).

REMARK 1. Formula (16) is proved in [10] in the case $K \cap \mathbb{Z}^{n}=\mathbb{Z}_{+}^{n}$, while in [11] for the intersection of $K$ with the sublattice $\Lambda$ of the lattice $\mathbb{Z}^{n}$ generated by some vectors $a^{1}, \ldots, a^{s}$, with $s \leq n$.

As an example of the applications of (15), consider some problem concerning generalized Dyck paths:

Find the number of paths $f\left(x_{1}, x_{2}\right)$ from $(0,0)$ to $\left(x_{1}, x_{2}\right)$ with the meshsizes $h_{1}=(1,1), h_{2}=(1,-1)$, and $h_{3}=(1,0)$.

It is obvious that $f\left(x_{1}, x_{2}\right) \neq 0$ only for $\left(x_{1}, x_{2}\right)$ lying in the rational cone

$$
K=\left\{\left(x_{1}, x_{2}\right) \in \mathbb{R}^{2}:\left(x_{1}, x_{2}\right)=\lambda_{1} h_{1}+\lambda_{2} h_{2},\left(\lambda_{1}, \lambda_{2}\right) \in \mathbb{R}_{+}^{2}\right\} .
$$

For the required number of paths $f\left(x_{1}, x_{2}\right)$ the difference equation is

$$
f\left(x_{1}+2, x_{2}\right)-f\left(x_{1}+1, x_{2}+1\right)-f\left(x_{1}+1, x_{2}-1\right)-f\left(x_{1}+1, x_{2}\right)=0,
$$

the set $\Omega$ consists of the points $\{(2,0),(1,1),(1,-1),(1,0)\}$, the characteristic polynomial $P(z, w)$ equals $z^{2}-z w-z w^{-1}-z$, the point $(2,0)$ satisfies $(6)$, and the Cauchy data are given on $X_{0}=\left\{x \in K \cap \mathbb{Z}^{2}\right.$ : $\underset{K}{x}(2,0)\}$. From the conditions of problem, it is easy to see that on $X_{0}$ the required function equals

$$
f(k, k)=f(k,-k)=1, \quad f(k+1, k)=f(k+1,-k)=k+1, \quad k=0,1,2, \ldots,
$$

while the generating function for the Cauchy data equals

$$
\Phi(z, w)=\frac{z}{z-w}+\frac{z}{(z-w)^{2}}+\frac{z w}{z w-1}+\frac{z w^{2}}{(z w-1)^{2}}+1+\frac{1}{z} .
$$

By Theorem 3 we find the generating function

$$
F(z, w)=\sum_{\left(x_{1}, x_{2}\right) \in K \cap \mathbb{Z}^{2}} \frac{f\left(x_{1}, x_{2}\right)}{z^{x_{1}} w^{x_{2}}}
$$

for the solution to $(17)-(18)$ :

$$
F(z, w)=\frac{z^{2}}{z^{2}-z w-z w^{-1}-z} .
$$

Observe that in this example the rationality of the generating function $\Phi(z, w)$ of the Cauchy data implies the rationality of the generating function $F(z, w)$ of the solution.

By analogy with Motzkin paths, we can ask for the number of paths crossing the line $q x_{1}-p x_{2}=0$, where $(p, q) \in K \cap \mathbb{Z}^{2}$. From the viewpoint of the theory of generating functions, we have to find the $(p, q)$-diagonal of the Laurent series $F(z, w)$. It is known (see [12]) that we can express the diagonal

$$
F_{(p, q)}(t)=\sum_{k=0}^{\infty} \frac{f(p k, q k)}{t^{k}}
$$

of the Laurent series of the rational function in integral form

$$
F_{(p, q)}(t)=\frac{1}{(2 \pi i)^{2}} \iint_{\Gamma} \frac{F(z, w)}{\left(1-\frac{z^{p} w^{q}}{t}\right)} \frac{d z}{z} \wedge \frac{d w}{w},
$$

and that it is algebraic. Here $\Gamma$ is the skeleton of the bicircle $\Gamma=\log ^{-1} u$, with $u \in E_{(2,0)}$, where $E_{(2,0)}$ is the component of the complement to the amoeba of the characteristic polynomial corresponding to the vertex $(2,0)$ of the Newton polytope $N_{P}$. For instance, the $(1,0)$-diagonal in this case equals

$$
F_{(1,0)}(t)=\frac{1}{(2 \pi i)^{2}} \iint_{\Gamma} \frac{z^{2}}{\left(z^{2}-z w-z w^{-1}-z\right)\left(1-\frac{z}{t}\right)} \frac{d z}{z} \wedge \frac{d w}{w}=\frac{t}{\sqrt{t^{2}-2 t-3}} .
$$




\section{D-Finite Series Supported on a Rational Cone}

For formal power series in one variable the concept of $D$-finiteness is studied systematically in $[6,13]$. For multiple power series this concept appears in [7].

Definition 2. A formal power series $\mathscr{F}(\xi) \in \mathbb{C}[[\xi]]$ is called $D$-finite whenever it satisfies a system of differential equations

$$
P_{k}^{i}(\xi) \frac{\partial^{k} \mathscr{F}}{\partial \xi_{i}^{k}}+\cdots+P_{1}^{i}(\xi) \frac{\partial \mathscr{F}}{\partial \xi_{i}}+P_{0}^{i}(\xi) \mathscr{F}=0, \quad i=1, \ldots, n,
$$

where $P_{j}^{i}(\xi)$ are polynomials.

One of the main properties of $D$-finite power series is that they constitute a subalgebra of the algebra of power series. In the ring $\mathbb{C}_{K}[[z]]$ of Laurent series the usual partial derivative $\frac{\partial}{\partial z_{i}}$ is not a derivation because for $x \in K \cap \mathbb{Z}^{n}$ the points $x+e^{i}$, where $e^{i}$ are the standard unit vectors, in general need not lie in $K \cap \mathbb{Z}^{n}$. This means that the $D$-finite Laurent series (5) in the sense of Definition 2 do not even constitute a subring of $\mathbb{C}_{K}[[z]]$. Therefore, instead of the partial derivatives, in Definition 1 we use the operators $D_{i}$, for $i=1, \ldots, n$, of the form (8). Let us establish a connection between Definitions 1 and 2 .

The main tool for that is the change of variables $\xi=z^{A}$, where $\xi_{i}=z_{1}^{a_{1}^{i}} \ldots z_{n}^{a_{n}^{i}}$ for $i=1, \ldots, n$. However, doing this in the series (5) directly, in general we obtain Puiseux series, and nothing is known about their $D$-finiteness properties. In this section we verify in Proposition 3 that we can express every Laurent series (5) as a sum of series each of which, upon the change of variables $\xi=z^{A}$ and up to multiplication by a monomial, becomes a $D$-finite power series.

Denote by

$$
\Lambda=\left\{x \in \mathbb{Z}^{n}: x=\lambda_{1} a^{1}+\cdots+\lambda_{n} a^{n}, \lambda_{i} \in \mathbb{Z}, \text { for } i=1, \ldots, n\right\}
$$

the sublattice of $\mathbb{Z}^{n}$ generated by the vectors $a^{1}, \ldots, a^{n}$. For every $v \in \mathbb{Z}^{n}$ denote by $\Lambda_{v}=v+\Lambda$ the shift of $\Lambda$ by the vector $v$. Considering the shifts of $\Lambda$ by vectors with integer coordinates lying in the parallelotope

$$
\Pi_{K}=\left\{x \in \mathbb{R}^{n}: 0 \underset{K}{\leq} x \underset{K}{<} \tau\right\}
$$

where $\tau=a^{1}+\cdots+a^{n}$, we show easily that $\bigcup_{v \in \Pi_{K} \cap \mathbb{Z}^{n}} \Lambda_{v}=\mathbb{Z}^{n}$. Therefore, we can uniquely express each series $F(z)=\sum_{x \in K \cap \mathbb{Z}^{n}} f(x) z^{-x}$ in the ring $\mathbb{C}_{K}[[z]]$ as the sum

$$
F(z)=\sum_{v \in \Pi_{K} \cap \mathbb{Z}^{n}} F_{v}(z)
$$

with

$$
F_{v}(z)=\sum_{x \in K \cap \Lambda_{v}} f(x) z^{-x}=z^{-v} \Theta_{v}(z)
$$

and $\Theta_{v}(z)=\sum_{y \in K \cap \Lambda} f(v+y) z^{-y}$. Observe that $\Theta_{v}(z)$ lies in the subring $\mathbb{C}_{K \cap \Lambda}[[z]]$ of $\mathbb{C}_{K}[[z]]$ consisting of the series supported by $K \cap \Lambda$.

Proposition 2. The series $F(z)$ and $F_{v}(z)$ related by (20) and (21) satisfy

$$
F_{v}(z)=\frac{1}{\Delta^{n}} \sum_{J} R^{a-v J} F\left(R^{J} z\right)
$$

where $R=\left(R_{1}, \ldots, R_{n}\right)$, with $R_{j} \neq 1$ for $j=1, \ldots, n$, are the roots of the system of equations

$$
R^{a^{i}}=1, \quad i=1, \ldots, n,
$$

and $J=\left(j_{1}, \ldots, j_{n}\right)$ with $1 \leq j_{1} \leq \Delta, \ldots, 1 \leq j_{n} \leq \Delta$, and $\Delta=\operatorname{det} A \neq 0$. 
Proof. We need to generalize the concept of multisection of a power series (see [14]). Recall that in the one-dimensional case the $k$ th $q$-section of the series $G(\xi)=\sum_{j=0}^{\infty} g(j) \xi^{j}$ is defined as

$$
G_{k}(\xi ; q)=\sum_{j=0}^{\infty} g(k+j q) \xi^{k+j q}, \quad k=0,1, \ldots, q-1,
$$

while every $k$ th $q$-section of a series can be expressed in terms of the original series as

$$
G_{k}(\xi ; q)=\frac{1}{q} \sum_{j=1}^{q} r^{q-k j} G\left(r^{j} \xi\right), \quad k=0,1, \ldots, q-1,
$$

where $r$ is a primitive degree $q$ root of 1 ; i.e., $r^{q}=1$ and $r \neq 1$.

Define the concept of multisection for multiple power series. Fix $q=\left(q_{1}, \ldots, q_{n}\right) \in \mathbb{Z}_{+}^{n}$ and consider the half-open parallelepiped $\Pi_{+}=\left\{x \in \mathbb{R}_{+}^{n}: 0 \leq x<q\right\}$. Denote by $k \in \Pi_{+} \cap \mathbb{Z}^{n}$ the points with integer coordinates; their number equals $\# \Pi_{+} \cap \mathbb{Z}^{n}=q_{1} \cdot \ldots \cdot q_{n}$. We can express the integer lattice $\mathbb{Z}^{n}$ as $\mathbb{Z}^{n}=\bigcup_{k \in \Pi_{+} \cap \mathbb{Z}^{n}}\left(k+q \mathbb{Z}^{n}\right)$, where the union is over all shifts of the sublattice $q \mathbb{Z}^{n}=\left(q_{1} \mathbb{Z}\right) \times \cdots \times\left(q_{n} \mathbb{Z}\right)$ by the vectors $k \in \Pi_{+} \cap \mathbb{Z}^{n}$.

Define the $k$ th $q$-section of the power series $\mathscr{F}(\xi)=\sum_{\lambda \in \mathbb{Z}_{+}^{n}} h(\lambda) \xi^{\lambda}$ as

$$
\mathscr{F}_{k}(\xi ; q)=\sum_{\lambda \in k+q \mathbb{Z}_{+}^{n}} h(\lambda) \xi^{\lambda}
$$

The following higher-dimensional analog of (23) holds:

$$
\mathscr{F}_{k}(\xi ; q)=\frac{1}{q_{1} \cdot \ldots \cdot q_{n}} \sum_{J} r^{q-k J} \mathscr{F}\left(r^{J} \xi\right)
$$

where $J=\left(j_{1}, \ldots, j_{n}\right)$ with $1 \leq j_{1} \leq q_{1}, \ldots, 1 \leq j_{n} \leq q_{n}$.

Indeed, replacing on the right-hand side of (24) the multiple sum by the repeated sum

$$
\frac{1}{q_{1}} \sum_{j_{1}=0}^{q_{1}} r_{1}^{q_{1}-k_{1} j_{1}} \ldots \frac{1}{q_{n}} \sum_{j_{n}=0}^{q_{n}} r_{n}^{q_{n}-k_{n} j_{n}} \mathscr{F}\left(r^{j_{1}} \xi_{1}, \ldots, r^{j_{n}} \xi_{n}\right)
$$

and applying (23) $n$ times, we obtain (24).

Return to the proof of (22). In the expression (20) for the series $F(z)$ make the change of variables $z^{-A}=\xi^{\Delta}$, where $z^{A}=\left(z_{1}^{a_{1}^{1}} \ldots z_{n}^{a_{n}^{1}}, \ldots, z_{1}^{a_{1}^{n}} \ldots z_{n}^{a_{n}^{n}}\right)$. Put $k=\Delta A^{-1} v$ and $q=\Delta A^{-1} \tau$ and observe that $q=(\Delta, \ldots, \Delta)$. Upon changing the index of summation $x=\frac{A \lambda}{\Delta}$ in the series $F_{v}(z)$ on the right-hand side of $(20)$, we obtain $\mathscr{F}(\xi)=\sum_{k} \mathscr{F}_{k}(\xi)$, where

$$
\mathscr{F}(\xi)=F\left(\xi^{-\Delta A^{-1}}\right), \quad \mathscr{F}_{k}(\xi)=\sum_{\lambda \in q \mathbb{Z}_{+}^{n}} f\left(\frac{A k+A \lambda}{\Delta}\right) \xi^{k+\lambda} .
$$

Since $\mathscr{F}_{k}$ is the $k$ th $q$-section of $\mathscr{F}$, by $(24)$ we have

$$
\mathscr{F}_{k}(\xi)=\frac{1}{\Delta \cdot \ldots \cdot \Delta} \sum_{J} r^{q-k J} \mathscr{F}\left(r^{J} \xi\right)=\frac{1}{\Delta^{n}} \sum_{J} r^{q-k J}\left(\sum_{\lambda \in q \mathbb{Z}_{+}^{n}} f\left(\frac{A \lambda}{\Delta}\right) r^{J \lambda} \xi^{\lambda}\right) .
$$

Returning to the variable $z$, we obtain

$$
F_{v}(z)=\frac{1}{\Delta^{n}} \sum_{J} R^{a-v J} \sum_{x \in K \cap \mathbb{Z}^{n}} f(x) R^{x J} z^{x}=\frac{1}{\Delta^{n}} \sum_{J} R^{\tau-v J} F\left(R^{J} z\right) .
$$


Remark 2. Proposition 2 implies that $F(z) \in \mathbb{C}_{K}[[z]]$ is $D$-finite if and only if so are all terms $F_{v}(z)$ in (20), while by (21) the $D$-finiteness of $F(z)$ is equivalent to the $D$-finiteness of all series $\Theta_{v}(z)$. Moreover, for the series in $\mathbb{C}_{K \cap \Lambda}[[z]]$ we may assume in Definition 1 that $Q_{i}^{j}(z) \in \mathbb{C}_{K \cap \Lambda}[z]$.

Given a mapping $\mathscr{A}$ from $\mathbb{Z}_{+}^{n}$ to $K \cap \Lambda$ as $\lambda \rightarrow A \lambda$, denote by $\mathscr{A}^{*}$ the mapping from the ring $\mathbb{C}_{K \cap \Lambda}[[z]]$ of Laurent series into the ring $\mathbb{C}[[\xi]]$ of power series induced by $\mathscr{A}$ as

$$
\mathscr{A}^{*}: \sum_{x \in K \cap \Lambda} f(x) z^{-x} \rightarrow \sum_{\lambda \in \mathbb{Z}_{+}^{n}} f(A \lambda) \xi^{\lambda}
$$

where $z^{-A}=\xi$.

Proposition 3. The series $\Theta(z) \in \mathbb{C}_{K \cap \Lambda}[[z]]$ is $D$-finite in the sense of Definition 1 if and only if the series $\mathscr{A}^{*}(\Theta) \in C[[\xi]]$ is $D$-finite in the sense of Definition 2.

Proof. Necessity: By assumption, $\Theta(z)$ satisfies (9). Upon changing the variables $z^{-A}=\xi$ and making standard rearrangements, we infer that $\mathscr{A}^{*}(\Theta)=\sum_{\lambda \in \mathbb{Z}_{+}^{n}} f(A \lambda) \xi^{\lambda}$ satisfies a system of the form (19). It is important to note that the change of the summation index $x \in K \cap \Lambda$ to $\lambda \in \mathbb{Z}_{+}^{n}$ is possible because $\mathscr{A}$ is bijective.

Sufficiency: Since $\mathscr{A}^{*}(\Theta)$ is $D$-finite in the sense of Definition 2, it satisfies a system of differential equations of the form (19). Reversing the argument in the proof of necessity, we infer that the series $\Theta(z)$ satisfies (9).

\section{Proof of Theorem 2}

Let us establish the two propositions that imply Theorem 2.

Proposition 4. If a series $F(z) \in \mathbb{C}_{K}[[z]]$ is $D$-finite (rational or algebraic) then

$$
F_{\omega}(z)=\sum_{x \in K \cap \mathbb{Z}^{n}, x \underset{K}{x}} f(x) z^{-x}
$$

is $D$-finite (rational or algebraic) for every $\omega \in K \cap \mathbb{Z}^{n}$.

Proof. By (20) and (21),

$$
F(z)=\sum_{v \in \Pi_{K} \cap \mathbb{Z}^{n}} z^{-v} \Theta_{v}(z)
$$

By the hypothesis of Proposition 4 and Remark 2 to Proposition 2, the series $\Theta_{v}(z)$ are $D$-finite (rational or algebraic). Upon changing the variables $z^{-A}=\xi$, instead of the Laurent series $\Theta_{v}$ we obtain the power series $\mathscr{A}^{*}\left(\Theta_{v}\right) \in \mathbb{C}[[\xi]]$, which are $D$-finite (rational or algebraic) series by Proposition 3. Hence, it suffices to justify Proposition 4 for $\mathscr{F}(\xi)$ in $\mathbb{C}[[\xi]]$. Use the property that the sections of a $D$-finite (rational or algebraic) power series are also $D$-finite (rational or algebraic).

We need the concept of $(n-1)$-dimensional section of a power series $\mathscr{F}(\xi)=\sum_{\lambda \in \mathbb{Z}_{+}^{n}} h(\lambda) \xi^{\lambda}$, which is defined as

$$
\mathscr{F}_{j}^{i}(\xi)=\sum_{x[i] \in \mathbb{Z}_{+}^{n-1}} h\left(\xi_{1}, \ldots, \xi_{i-1}, j, \xi_{i+1}, \ldots, \xi_{n}\right) \xi[i]^{x[i]},
$$

where $[i]$ indicates the absence of the $i$ th variable.

At the first step consider the difference

$$
\mathscr{X}(\xi)=\mathscr{F}(\xi)-\sum_{k=0}^{\omega_{1}-1} \xi^{k} \mathscr{F}_{k}^{1}(\xi) .
$$


Its coefficients for all powers $\xi^{\lambda}$ with $0 \leq \lambda_{1} \leq \omega_{1}-1$ vanish, and for the remaining powers they are equal to $h(\lambda)$. Since the sections of $\mathscr{F}_{k}^{1}$ are $D$-finite, while the $D$-finite series constitute an algebra, $\mathscr{X}(\xi)$ is a $D$-finite series. At the second step consider the difference $\mathscr{X}(\xi)-\sum_{k=0}^{\omega_{2}-1} \xi^{k} \mathscr{X}_{k}^{2}(\xi)$ and, successively repeating this procedure for the remaining variables, infer that $\sum_{\lambda \geq \omega} h(\lambda) \xi^{\lambda}$ is a $D$-finite (rational or algebraic) series. Consequently, so is

$$
\mathscr{F}_{\omega}(\xi)=\mathscr{F}(\xi)-\sum_{\lambda \geq \omega} h(\lambda) \xi^{\lambda} .
$$

This is also true for the series $\mathscr{A}^{*}\left(\Theta_{\omega, v}\right) \in \mathbb{C}[[\xi]]$, which upon passing to the variables $z$ become $D$-finite (rational or algebraic) by Proposition 3. This implies that the series

$$
F_{\omega}(z)=\sum_{v} z^{-v} \Theta_{\omega, v}(z)
$$

is $D$-finite (rational or algebraic).

Proposition 5. The product of $D$-finite Laurent series in $\mathbb{C}_{K}[[z]]$ is $D$-finite.

Proof. Take two $D$-finite Laurent series $U(z), W(z) \in \mathbb{C}_{K}[[z]]$. Using (20) and (21) for them, we deduce that the product $U(z) W(z)$ is a linear combination of the products $z^{-v-q} \Theta_{v} \Theta_{q}$, where $\Theta_{v}(z), \Theta_{q}(z) \in \mathbb{C}_{K \cap \Lambda}[[z]]$. Upon setting $z^{-A}=\xi$ in the series $\Theta_{v}(z)$ and $\Theta_{q}(z)$, we obtain two power series $\Theta_{v}\left(\xi^{A^{-1}}\right), \Theta_{q}\left(\xi^{A^{-1}}\right) \in \mathbb{C}[[\xi]]$, which are $D$-finite by Proposition 3 . Therefore, their product is also $D$-finite (see [7]). Returning to the variable $z$ and using Proposition 3 again, we validate Proposition 5.

Proof of Theorem 2. Use (15), which relates the generating function for the solution $F(z)$ to problem (3)-(4) and the series $\Phi_{\omega}$ constructed from the Cauchy data (4). By Proposition 4 , the $D$ finiteness (rationality or algebraicity) of the generating function for the Cauchy data $\Phi_{m}$ implies the same property of $\Phi_{\omega}$ for all $\omega \in \Omega$. The rationality and algebraicity of the product $c_{\omega} z^{\omega} \frac{1}{P(z)} \Phi_{\omega}(z)$ is obvious, while its $D$-finiteness follows from Proposition 5 .

REMARK 3 . We should mention that Theorem 2 in the case of generating functions in $\mathbb{C}_{K \cap \Lambda}[[z]]$ was considered in [15].

\section{References}

1. Samarskiu A. A., The Theory of Difference Schemes [in Russian], Nauka, Moscow (1977).

2. Duffin R. J., "Potential theory on rhombic lattice," J. Comb. Theory, 5, 258-272 (1968).

3. Zeilberger D. A., "New basis for discrete analytic polynomials," J. Austral. Math. Soc. Ser. A, 23, 95-104 (1977).

4. Danilov O. A. and Mednykh A. D., "Discrete analytic functions in several variables and the Taylor formula," Vestnik NGU. Ser. Mat. Mekh. Inform., 9, No. 2, 38-46 (2009).

5. Bousquet-Mélou M. and Petrovšek M., "Linear recurrences with constant coefficients: the multivariate case," Discrete Math., 225, 51-75 (2000).

6. Stanley R. P., Enumerative Combinatorics, Cambridge Univ. Press, Cambridge (1999).

7. Lipshitz L., "D-Finite power series," J. Algebra, 122, No. 2, 353-373 (1989).

8. Hörmander L., An Introduction to Complex Analysis in Several Variables [Russian translation], Mir, Moscow (1968).

9. Forsberg M., Passare M., and Tsikh A., "Laurent determinants and arrangements of hyperplane amoebas," Adv. Math., 151, 45-70 (2000).

10. LeInartas E. K., "Stability of the Cauchy problem for a multidimensional difference operator and the amoeba of the characteristic set," Sib. Math. J., 52, No. 5, 864-870 (2011).

11. Nekrasova T. I., "Cauchy problem for multidimensional difference equations in lattice cones," J. Sib. Fed. Univ. Math. Phys., 5, 576-580 (2012).

12. Pochekutov D. Yu., "Diagonals of the Laurent series of rational functions," Sib. Math. J., 50, 1081-1090 (2009).

13. Stanley R., "Differentiably finite power series," Eur. J. Comb., No. 1, 175-188 (1980).

14. Riordan J., Combinatorial Identities, John Wiley and Sons, New York etc. (1968).

15. Nekrasova T. I., "On the hierarchy of generating functions for solutions of multidimensional difference equations," Izv. Irkutsk Gos. Univ. Ser. Matematika, 9, 91-103 (2014).

E. K. LeǏnARTAS; T. I. Nekrasova

Siberian Federal University, Krasnoyarsk, Russia

E-mail address: lein@mail.ru; t.neckrasova@gmail.com 